\title{
Heat Transfer Enhancement by Flow Control in a Rectangular Horizontal Channel
}

\author{
Ghazali Mebarki, Samir Rahal, and Abdelhek Hamza
}

\begin{abstract}
Laminar fluid flows and heat transfers, by forced convection, in a rectangular horizontal channel, have been numerically investigated. Three blocks, simulating electronic components, have been attached to the channel bottom wall. In order to control the flow and enhance the heat transfer rate, a rectangular cross section bar, acting as a vortex generator, has been attached to the channel top wall. The Navier-Stokes and energy equations are solved using FLUENT. Velocity and temperature fields as well as local Nusselt numbers have been obtained for various imposed conditions. Our numerical simulation procedure has been validated by comparison with results of other authors. The obtained results indicate that thanks to the control element, heat transfer in the channel is enhanced. For each block, the best position of the vortex generator, which allows maximal heat dissipation, is when the control element is located above the considered block.
\end{abstract}

Index Terms-Electronic cooling, heat transfer enhancement, nusselt number, vortex generator.

\section{INTRODUCTION}

The improvement of device performance such as electronic component cooling systems, compact heat exchangers, solar energy systems, biomedical devices etc..., is of great interest to researchers and is intensively studied recently. Indeed, the tendency for more compactness, economy in manufacturing and operating costs and energy consumption, has become the rule in the design of the above mentioned systems. To avoid temperature rises, which induce overheating of components that can be detrimental to electronic devices, better cooling allowing maximal heat dissipation is necessary. For that purpose, a promising technique is to introduce obstacles in the flow, known as vortex generators, which act as flow control elements. Vortex generators allow passively fluid mixing and thereby enhance the heat transfer with a low additional pressure loss. Recently, vortex generators have been used by many researchers for the heat transfer enhancement in various thermal systems. For example, [1] numerically studied the effect of longitudinal vortex generator on the heat transfer in a fin-and-tube heat exchanger. The results reveal that the transverse flow of air stream through the punched holes disturbs the air flow in the lower channel, enhancing the heat transfer on the under surface of fin. Reference [2] proved that the use of a triangular prism could enhance significantly the heat transfer in a channel. Reference [3] obtained numerically the rate of heat transfer enhancement in a

Manuscript received January 4, 2013; revised March 4, 2013.

Ghazali Mebarki and Samir Rahal are with LESEI laboratory, Department of Mechanical Engineering, Faculty of Technology, University of Batna, Algeria (e-mail: g.mebarki@yahoo.fr; rahal.samir16@gmail.com). channel due to the presence of a triangular element. The results indicate that heat transfer in the channel is augmented by around $15 \%$. Turbulent flow and heat transfer in a heated channel with a triangular prism has been investigated, numerically by [4]. The results showed larger heat transfer augmentation. The control of laminar steady forced convection heat transfer in a channel, with three blocks and a triangular adiabatic control element, has been studied numerically by [5]. It has been shown that the heat transfer is enhanced and the best element position determined. Reference [6] conducted a numerical study to analyse the unsteady flow and heat transfer in a horizontal channel with a built-in heated cylinder. The heat transfer was found to be slightly affected by the blockage ratio and correlations for the Nusselt number were obtained. Heat transfer and fluid flow characteristics in a channel, with the presence of a triangular prism, has been numerically investigated in the laminar flow regime by [7]. It has been found that the average Nusselt number is augmented and the heat transfer increases with the blockage ratio. Heat transfer enhancement for triangular dual prisms has been found to be larger than that for the case of a single triangular prism, for the same blockage ratio. Reference [8] studied the fluid flow and heat transfer across a long equilateral triangular cylinder set in a horizontal channel for a fixed blockage ratio of 0.25 . It has been found that the average Nusselt number increases with the Reynolds number. Simple correlations for Nusselt numbers have also been obtained. Two dimensional laminar forced convection heat transfers around a horizontal triangular cylinder in an air flow have been investigated numerically by [9]. Two orientations of the triangular cylinder have been considered, the first corresponds to the case for which the vertex of the triangle is facing the flow. As for the second case, the base of the triangle is facing the flow. Correlations are obtained and local Nusselt numbers have been found to be in qualitative agreement with corresponding data reported in the literature. Reference [10] analyzed the effect of wall proximity of a triangular cylinder on the heat transfer and flow in a horizontal channel. Results showed that when the triangular element is close to the wall, the vortex shedding is removed and subsequently the heat transfer rate decreases at low Reynolds number. Experimental investigations have been reported by [11] on steady forced convection heat transfer from the outer surfaces of horizontal triangular cylinders in an air flow. Local Nusselt numbers around the obstacles are observed to decrease, at the beginning, up to the separation points and then increase, in the transition regime, up to the turbulent limit where they decrease again. Reference [12] studied the heat transfer and fluid flow in a channel using an inclined block as an obstacle. By the use of the inclined 
block, larger vortices were produced and thus heat transfer was augmented considerably. A heat transfer optimisation of a channel with three blocks attached to its bottom wall and an inserted triangular cylinder has been carried out by [13]. The goal of the study is to maximize the heat transfer rate as well as achieving heat flux uniformity above the blocks. A genetic algorithm combined with a Gaussian process has been used as an optimisation algorithm for that purpose. The results showed that the larger value of the standard deviation multiplier is the more uniform Nusselt numbers are. Moreover, the optimum position of the vortex generator has been found to be above the first block.

In this study we present a numerical simulation of flow and heat transfer by forced convection in a horizontal channel with three blocks attached to its bottom wall. The blocks simulate electronic components. In order to enhance heat transfer, a rectangular cross section control element, acting as a vortex generator, has been attached to the channel top wall. The control element best position, allowing maximal heat dissipation, has been determined.

\section{GEOMETRY AND GOVERNING EQUATIONS}

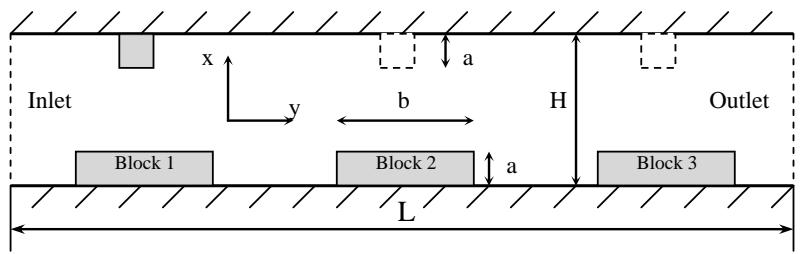

Fig. 1. Geometry of the channel with blocks and the control element.

The geometry of the studied problem is shown in Fig. 1. It consists of a 2-D horizontal plane channel of $20 \mathrm{~cm}$ length $(L)$ and $1 \mathrm{~cm}$ height $(H)$, with three blocks, simulating electronic components, attached to its bottom surface. An adiabatic square element of $0.25 \mathrm{~cm}$ side $(a)$ is also attached to the channel top surface. Each block is $0.25 \mathrm{~cm}$ height $(a)$ and 2 $\mathrm{cm}$ length $(b)$. Both top and bottom walls are assumed to be adiabatic. The fluid enters to the channel with uniform velocity and temperature. The governing two-dimensional equations, in a cartesian coordinate system, for incompressible, steady, laminar flow, with constant fluid properties, are as follows:

Continuity:

$$
\partial u / \partial x+\partial v / \partial y=0
$$

Momentum equation in $x$-direction:

$$
\begin{gathered}
u(\partial u / \partial x)+v(\partial u / \partial y)=-(1 / \rho)(\partial p / \partial x) \\
+v\left(\partial^{2} u / \partial x^{2}+\partial^{2} u / \partial y^{2}\right)
\end{gathered}
$$

Momentum equation in $y$-direction:

$$
\begin{gathered}
u(\partial v / \partial x)+v(\partial v / \partial y)=-(1 / \rho)(\partial p / \partial y) \\
+v\left(\partial^{2} v / \partial x^{2}+\partial^{2} v / \partial y^{2}\right)
\end{gathered}
$$

Energy equation:

$$
u(\partial T / \partial x)+v(\partial T / \partial y)=\alpha\left(\partial^{2} T / \partial x^{2}+\partial^{2} T / \partial y^{2}\right)
$$

where $u, v, p, T, \rho, v$ and $\alpha$ denote the velocity components, pressure, temperature, density, cinematic viscosity and thermal diffusivity respectively.

The boundary conditions are specified as follows:

At the channel inlet, $u=U_{0}, v=0$ and $T=T_{0}$.

On the rectangular control element and at the top and bottom walls, $u=v=0$ and $\partial T / \partial n=0$.

On the blocks, $u=v=0$ and $q=q_{0}$.

$U_{0}$ and $T_{0}$ are velocity and temperature at the inlet channel, $\mathrm{n}$ is the normal direction to the surface and $q_{0}$ is the heat flux generated by each block.

At the exit channel, the default outflow boundary condition in FLUENT is used, which assumes a zero diffusion flux for all flow variables. This is similar to Neumann boundary condition as: $\partial u / \partial x=\partial v / \partial x=\partial T / \partial x=0$

The inlet Reynolds number is calculated by the relation:

$$
\operatorname{Re}=U_{0} H / v
$$

The local Nusselt number is calculated by:

$$
N u=h H / k
$$

With: $(h)$ is the heat transfer coefficient and $(k)$ the fluid thermal conductivity.

\section{NUMERICAL PROCEDURE}

The grid generated by Gambit is shown in Fig. 2. The mesh has been refined in some regions such as around the blocks and the control element as well as along the channel bottom wall. Numerical simulations have been carried out with a number of 8232 elements. This number has been chosen thanks to a mesh independence study which showed that above this value the results do not depend on the number of elements any more. To resolve the governing equations, FLUENT has been used, which is based on the finite volume method. Second order upwind scheme has been used for the discretization of motion and energy equations. SIMPLE algorithm has been used for the pressure-velocity coupling.

The heat transfer coefficient and Nusselt number are calculated by Fluent on the basis of a constant reference temperature. This assumption is valid for external flows. However, for internal flows, as in our case, the reference temperature cannot be considered as constant. Therefore results given by Fluent are not suitable and the calculation of an average temperature of the mixture formula becomes necessary.

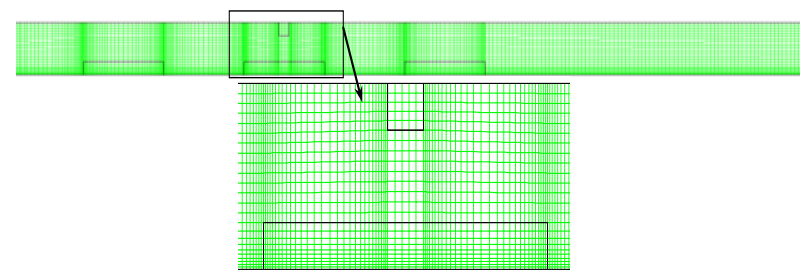

Fig. 2. Geometry and mesh of the studied problem. 
The local heat transfer coefficient is calculated by:

$$
q_{w}=h\left(T_{w}-T_{b u l k}\right)
$$

$q_{w}$ and $T_{w}$ are respectively the wall heat flux and temperature. $T_{\text {bulk }}$ is the bulk temperature calculated as follows:

$$
T_{\text {bulk }}=\int_{A} u T d A / \int_{A} u d A
$$

The channel section above the block is divided into several sections as shown in Fig. 3. In each section, wall heat flux and temperature are calculated.

\section{RESUlTS AND Discussions}

The working fluid is air with a Prandtl number $\operatorname{Pr}=0.71$. Various values of the Reynolds number have been considered. The heat flux generated by each block has been taken equal to $1000 \mathrm{~W} / \mathrm{m}^{2}$. Before presenting the results of this study, a validation of the numerical procedure has been conducted.

\section{A. Validation}

The numerical procedure validation has been conducted by considering the calculated Nusselt numbers for various Reynolds number. Both cases of a configuration with or without a control element have been considered. The average Nusselt number for the laminar regime has been calculated by the kakaç correlation [14] witch is given for a rectangular cross section channel, with an aspect ratio $(\alpha)$ and for an imposed wall heat flux by:

$$
\begin{aligned}
N u= & 8.235\left(1-10.6044 \alpha+61.1755 \alpha^{2}\right. \\
& \left.-155.1803 \alpha^{3}+176.9203 \alpha^{4}-72.9236 \alpha^{5}\right)
\end{aligned}
$$

A comparison between local Nusselt numbers for the three blocks and the average Nusselt number given by the kakaç [14] correlation is shown in Fig. 4, for Reynolds numbers equal to 342,479 or 547 . It is clear that the value of the average Nusselt number is very close to that of the local Nusselt calculated for the three blocks for the completely developed flow region (at the end of the block). It can also be noticed that Nusselt number decreases for the second and third blocs. Consequently, the closest block to the channel entrance will be better cooled.

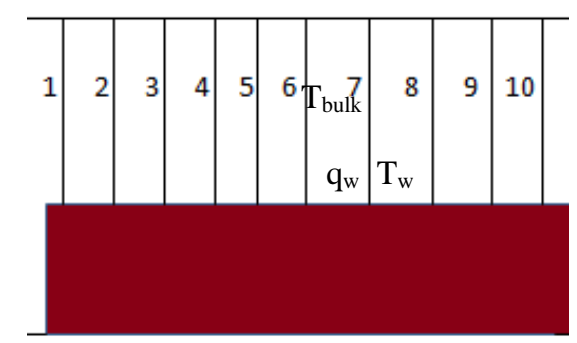

Fig. 3. Channel section subdivision for local Nusselt number calculation.
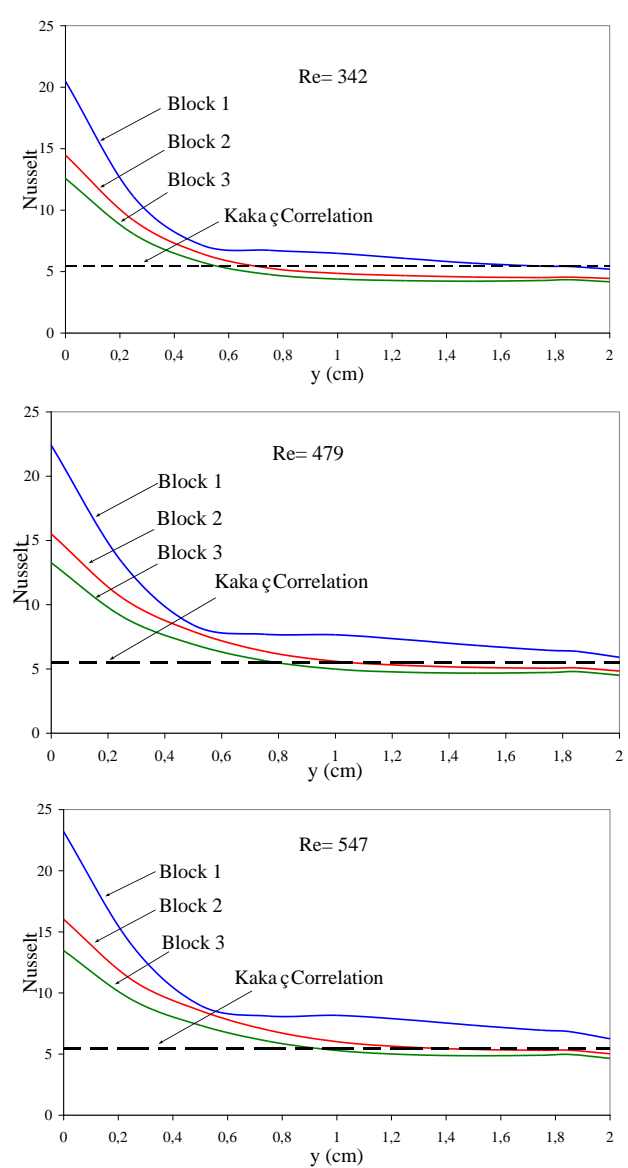

Fig. 4. Comparisons between calculated Nusselt number and kakaç correlation for different Reynolds numbers.

\section{B. Heat Transfer Control}

Results showing the effect of the control element on the fluid flow and heat transfer are presented in this section. The inlet fluid conditions at the entrance of the channel are: a uniform velocity corresponding to a Reynolds number equal to 479 and a constant temperature equal to $T_{0}=20^{\circ} \mathrm{C}$. The heat flux generated by each block is equal to $1000 \mathrm{~W} / \mathrm{m}^{2}$. The flow structure is specified with velocity magnitude contours and streamlines, while the heat transfer is characterized by temperature contours and the Nusselt number. In Fig. 5 and 6, contours of velocity magnitude and streamlines around the first block are represented for a channel without blocks and $\mathrm{Re}=479$. A strong flow circulation occurred behind each block. The largest velocity is observed far from the blocks, while the lowest velocity is observed around the blocks. This fact gives an explanation to the observed weak heat dissipation and consequently the heat transfer coefficient in this region is low. Fig. 7 shows the temperature contours in the channel. It is obvious that the cooling rate depends on the position of the block from the channel entrance. The closest block to the entrance is the most cooled. Local Nusselt numbers as functions of the Reynolds number, for each block, are shown in Fig. 8. The Nusselt number increases with the Reynolds number, which is in agreement with results obtained by other authors [5]-[9]. Fig. 9 shows the velocity magnitude contours and velocity vectors for a channel with the control element located above the first block for $\mathrm{Re}=$ 479. It can be noticed that the flow is accelerated due to the section contraction between the control element and the first block. 


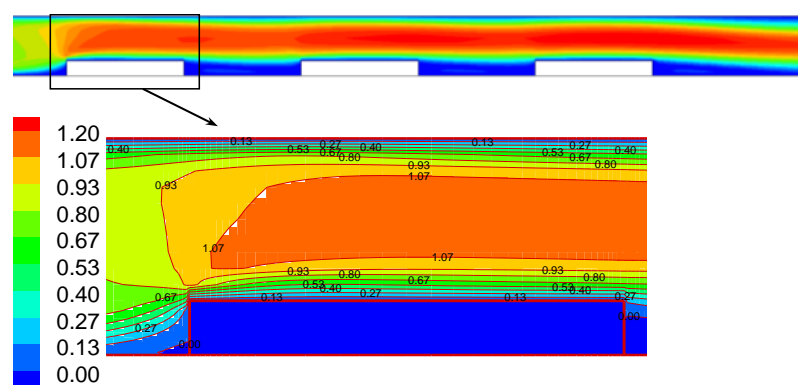

Fig. 5. Contours of velocity magnitude for a channel without the control element. $\mathrm{Re}=479$.

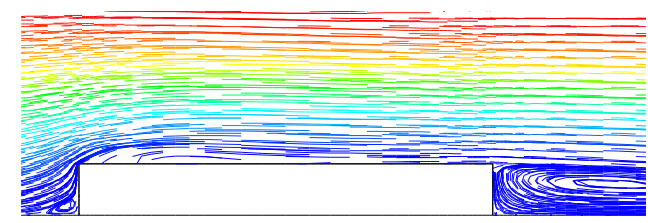

Fig. 6. Streamlines around the first block. $\mathrm{Re}=479$.
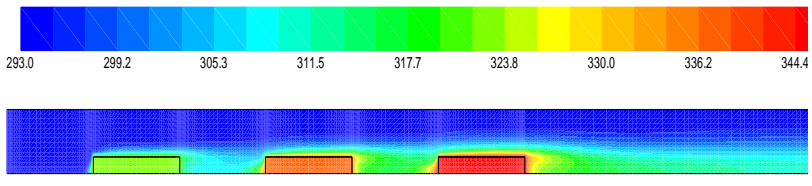

Fig. 7. Contours of static temperature for a channel without the control element. $\mathrm{Re}=479$.
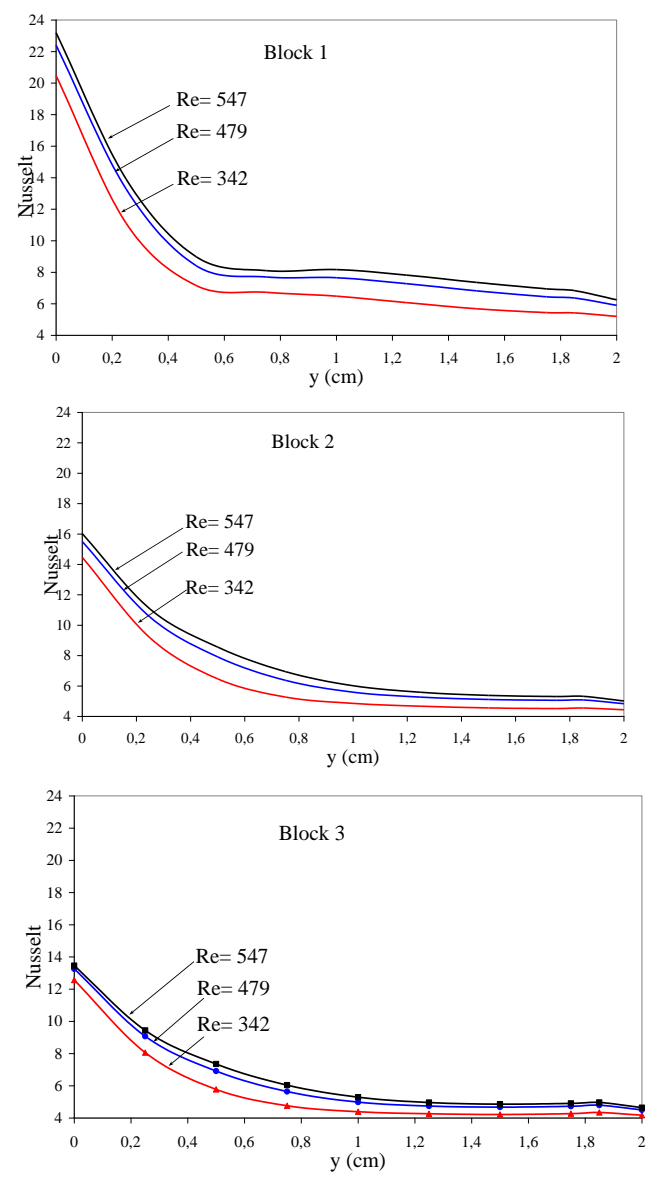

Fig. 8. Local Nusselt number for various Reynolds number corresponding to different blocks.

In Fig. 10, the velocity variations as functions of $(x)$, at the middle of the block, for two cases (with and without the control element), are shown. It is clear that the velocity is increased by the presence of the control element. Due to the flow acceleration, the first block is better cooled. This is clearly seen by observing the temperature contours in Fig. 11 and the temperature profile shown in Fig. 12.

When the element control is located above the second block or the third one (Fig. 13), its effect, on the velocity distribution along the upper surfaces of the blocks, can clearly be seen. The flow acceleration caused by the presence of the control element allows a better heat dissipation. This is confirmed by the temperature contours in Fig. 14 and the temperature profile of Fig. 15. It is obvious clearly that the presence of the control element above the blocks deceases significantly their temperatures. The heat transfer is therefore significantly enhanced by the presence of the control element. This is clearly seen in Fig. 16. For each block, the best position of the control element, which allows the best heat dissipation, corresponds to that located above the considered block. The other positions of the control element (above the other blocks) have a less influence on the heat transfer enhancement.

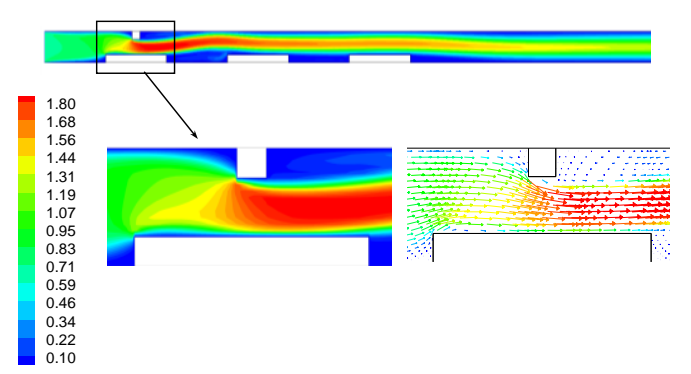

Fig. 9. Velocity magnitude contours and velocity vectors for a channel with the control element located above the first block. $\mathrm{Re}=479$

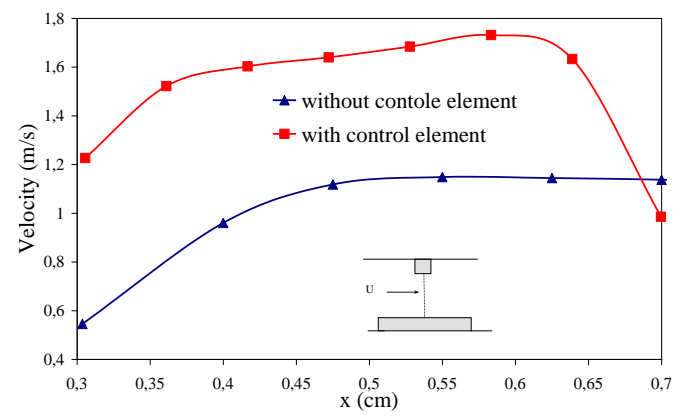

Fig. 10. Velocity profile at the middle of the first block for a channel with and without the control element. $\operatorname{Re}=.479$.

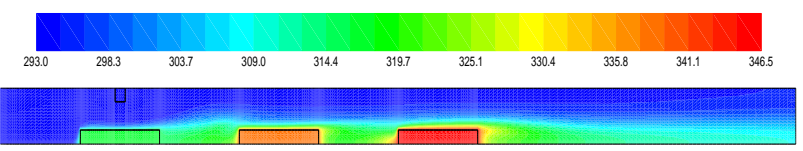

Fig. 11. Temperature contours for a channel with the control element located above the first block. $\mathrm{Re}=479$.

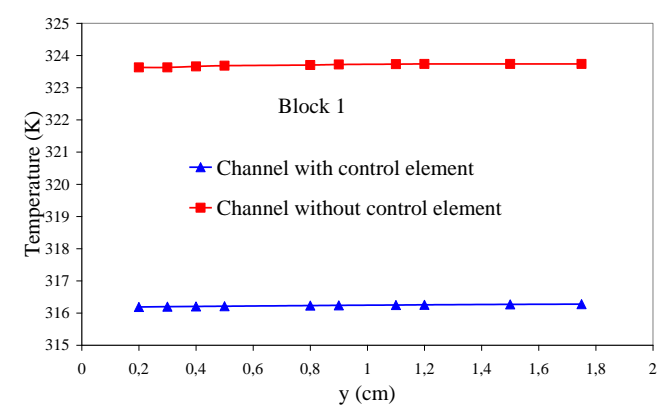

Fig. 12. Temperature along the upper surface of the first block. Two cases are shown (with and without the control element). $\mathrm{Re}=479$. 


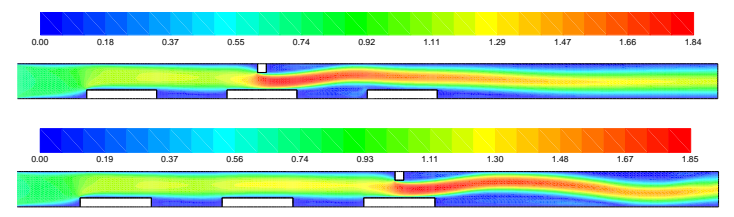

Fig. 13. Velocity magnitude contours in a channel with the control element located above the second block or the third one. $\mathrm{Re}=479$.

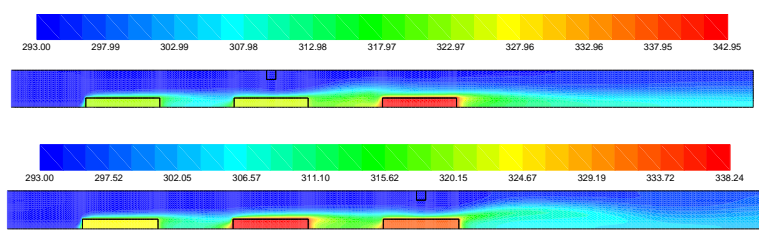

Fig. 14. Temperature contours in a channel with the control element located above the second block or the third one. $\operatorname{Re}=479$.
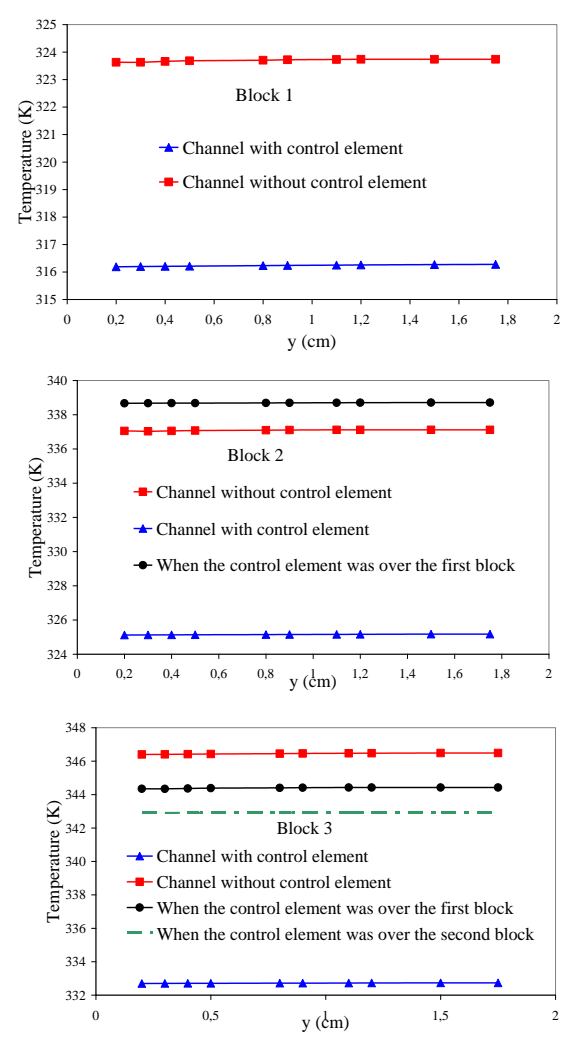

Fig. 15. Temperature profiles along the upper surface of $2^{\text {nd }}$ and $3^{\text {rd }}$ block. Re $=479$. Two cases (with and without the control element) are shown.
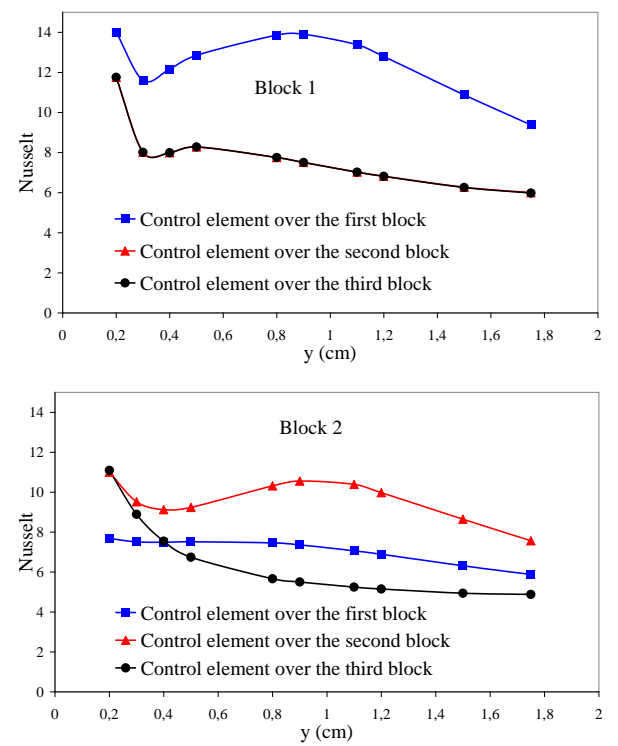

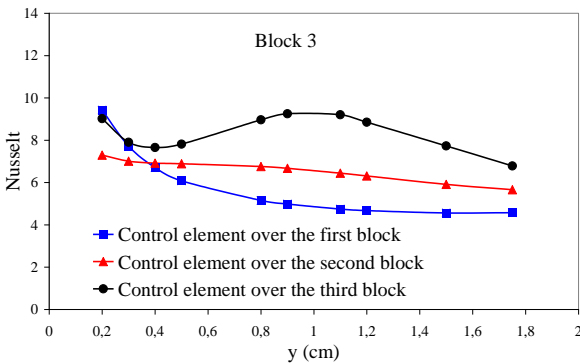

Fig. 16. Nusselt Number profiles corresponding to various positions of the control element. Above different block. $\mathrm{Re}=479$.

\section{CONCLUSION}

In this study, a numerical simulation of fluid flow and heat transfer by laminar forced convection, in a rectangular channel containing three blocks, has been carried out. Local Nusselt numbers, velocity and temperature contours are presented in order to describe the flow and heat transfer around the blocks. The validation of our numerical simulation procedure has been achieved by comparing our results with the Kakaç correlation, with which a fair agreement has been observed. As expected, the Nusselt number has been found to increase with Reynolds number. Heat transfer was found to be enhanced by a rectangular control element attached to the channel top wall. Indeed, the obtained results showed that the presence of the control element significantly enhances the heat dissipation and consequently reduces the temperatures of the blocks, which simulate electronic components. For each block, the best position of the control element, that allows the maximal heat dissipation, is when it is located above the considered block.

\section{REFERENCES}

[1] J. M. Wu and W. Q. Tao, "Effect of longitudinal vortex generator on heat transfer in rectangular channels," Applied Thermal Engineering, vol. 37, pp. 67-72, 2012.

[2] H. Abbassi, S. Turki, and S. Ben Nasrallah, "Numerical investigation of forced convection in a horizontal channel with a built-in triangular prism," Int. J. Thermal Sciences, vol. 40, pp. 649-658, 2001.

[3] H. Chattopadhyay, "Augmentation of heat transfer in a channel using a triangular prism,” Int. J. Thermal Sciences, vol. 46, pp. 501-505, 2007.

[4] A. C. Benim, H. Chattopadhyay, and A. Nahavandi, "Computational analysis of turbulent forced convection in a channel with a triangular prism," Int. J. Thermal Sciences, vol. 50, pp. 1973-1983, 2011.

[5] H. F. Oztop, Y. Varol, and D. E. Alnak, "Control of heat transfer and fluid flow using a triangular bar in heated blocks located in a channel," Int. Comm. in Heat and Mass Transfer, vol. 36, pp. 878-885, 2009.

[6] S. Turki, H. Abbassi, and S. B. Nasrallah, "Two-dimensional laminar fluid flow and heat transfer in a channel with a built-in heated square cylinder," Int. J. Thermal Sciences, vol. 42, pp. 1105-1113, 2003.

[7] B. Budania and H. Shergill, "Simulation Heat Transfer Enhancement in a Laminar Channel Flow with Built-in Triangular Prism," Int. Journal on Emerging Technologies vol. 3, no. 1, pp. 92-96, 2012.

[8] S. Srikanth, A.K. Dhiman, and S. Bijjam, "Confined flow and heat transfer across a triangular cylinder in a channel," International Journal of Thermal Sciences, vol. 49, pp. 2191-2200, 2010.

[9] O. Zeitoun, M. Ali, and A. Nuhait, "Convective heat transfer around a triangular cylinder in an air cross flow," International Journal of Thermal Sciences, vol. 50, pp. 1685-1697, 2011.

[10] M. Farhadi, K. Sedighi, and A. M. Korayem, "Effect of wall proximity on forced convection in a plane channel with a built-in triangular cylinder," Int. J. Thermal Sciences, vol. 49, pp. 1010-1018, 2010.

[11] M. Ali, O. Zeitoun, and A. Nuhait, "Forced convection heat transfer over horizontal triangular cylinder in cross flow," International Journal of Thermal Sciences, vol. 50, pp. 106-114, 2011. 
[12] M. Gupta, U. Khod, and S. Kumar, "Heat Transfer Augmentation Using an Inclined Block in Laminar Channel Flow," Recent Trends in Engineering Research, vol. 1, no.1, pp. 105-108, Dec. 2011.

[13] S. A. Beig, E. Mirzakhalili, and F. Kowsari, "Investigation of optimal position of a vortex generator in a blocked channel for heat transfer enhancement of electronic chips," International Journal of Heat and Mass Transfer, vol. 54, pp. 4317-4324, 2011.

[14] S. Kakaç, Boiler Evaporators and Condensers, New York U.S.A.: John wiley \& Sons Inc., 1991.

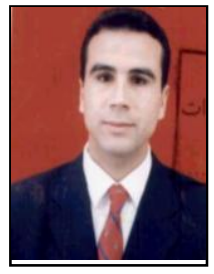

G. Mebarki was born in Algeria in 1969. Received the Dipl.-Ing. in mechanical engineering from the University of Batna Algeria in 1992, and then received the Magister Dipl. in Mechanical Engineering from the University of Batna Algeria in 1997. Obtained a two years training intership in GRESPI laboratory, University of Reims France in 2010; in order to prepare a Thesis in the field of heat transfer in mini and microchannels. He was an Assistant Teacher in Mechanical Engineering Department, University of Batna Algeria since 1998. 